\title{
Lumbar puncture training using simulation-based educational strategies. Experience in a clinical pediatric residency
}

\author{
Juan C. Vassallo, M.D. ${ }^{a}$, Bárbara Gouguenheim, M.D. ${ }^{a}$, Analía Ghiglione, M.D. ${ }^{a}$, \\ Nélida Bravo, B.S. ${ }^{a}$, Carla I. Prudencio, B.S. ${ }^{a}$, Florencia Villois, M.D. ${ }^{a}$, \\ Yamila Abadie, M.D. ${ }^{a}$, Ana Zubieta, M.D. ${ }^{a}$, Carol Golini, M.D. ${ }^{a}$, \\ Victoria Villar, M.D. ${ }^{a}$, and Susana P. Rodríguez, M.D. ${ }^{a}$
}

\begin{abstract}
Pediatricians should acquire multiple skills during their professional training, including procedural skills. Skill acquisition requires knowledge on theoretical bases, direct observation and, lastly, supervised repetitive practice. Training using simulators allows to learn procedures in a controlled setting, ensuring patients' safety, integrating this as a learning stage prior to the actual contact with patients. Here we report on the teaching experience of a simulated lumbar puncture procedure.

Training was provided to 112 first year pediatric residents who entered Hospital Prof. Dr. Juan P. Garrahan in the 2013-2014 period. Educational contents included communication with parents regarding the procedure, material preparation, compliance with biosafety standards, sepsis and general patient care, puncture and subsequent cerebrospinal fluid collection, and specimen collection.

Strategies included, in a sequential order, the introduction of theoretical aspects using the bibliography and audiovisual resources available at the hospital's online campus and subsequent practice of lumbar puncture in a 3-month-old infant phantom on a lateral recumbent position that allowed to make a puncture and collect cerebrospinal fluid. At each training session, the level of confidence was measured before and after the procedure, and a checklist was developed to verify an adequate compliance with each step of the procedure.

The simulated lumbar puncture training model has been introduced as an educational strategy of our Pediatric Residency Program.

Key words: lumbar puncture, medical training, simulation, pediatrics.
\end{abstract}

http:/ / dx.doi.org/10.5546/aap.2015.eng.544

E-mail Address:

Juan C. Vassallo, M.D.: jcvassallo@intramed.net

Funding:

Fundación Garrahan provided funds for the development of this study.

Conflict of Interest: None.

Received: 1-10-2015 Accepted: 5-8-2015 there is no chance of making modifications or monitoring advances; the chance of performing a lumbar puncture is random and, sometimes, sporadic. The advantages of using clinical simulations in professional training have been increasingly recognized. ${ }^{1}$ Simulation-based training allows to learn procedures in a controlled setting, ensuring patient safety, and integrating this as a learning stage prior to actual contact with patients. ${ }^{2,3}$

At the residency program of our hospital, we have prioritized those practices that, given their modality, frequency or impact, may be especially optimized by simulation strategies. Among these practices, lumbar puncture (LP) training was selected because it is one of the procedures to be performed by pediatric residents, either in the E.R. Department or during the hospitalization period. There is evidence that newly graduated physicians have little knowledge, a low level of confidence in their own skills and are not prepared to perform an LP in a child, ${ }^{4}$ although this has been identified as a mandatory skill by both national $l^{5}$ and international ${ }^{6}$ training programs.

Based on supplementary educational strategies that integrate training through an online platform and supervised simulated practices, first year pediatric residents (PR1) acquire and develop skills in a controlled and supervised setting before being in actual contact with children, in a standardized and safe manner for both patients and 
physicians. Given that this is a novel educational strategy, we decided to report on its development and mechanisms used for assessment from the perspective of both teachers and residents themselves. In addition, during a simulated and contextualized practice, communication between residents and parents regarding the procedure was also assessed.

The objective of this study is to report on the teaching experience with simulated lumbar puncture procedures at Hospital de Pediatría "Prof. Dr. Juan P. Garrahan".

Participants were PR1 who entered the hospital in the first three months of the hospital's program, between June and September of 2013 and 2014. Training was provided to all PR1 as part of the curricula. Authorization to publish the report was requested and personal data were safeguarded. The study was approved by the Institutional Review Committee.

\section{Educational strategy for lumbar puncture training and assessment}

First of all, educational contents were established. These included communication with parents regarding the need to perform a lumbar puncture, material preparation, compliance with biosafety standards, sepsis and patient general care (position, monitoring, analgesia), puncture and collection of cerebrospinal fluid, and specimen collection. The strategy was conducted sequentially, and included 1) introduction of theoretical aspects by reading specific bibliography and using audiovisual resources available at the hospital's online campus, and 2) LP practice and assessment using a phantom (Figure 1). A 3-month-old infant phantom on a lateral recumbent position with realistic anatomical landmarks was used for simulation. Spinal canal puncture allowed to obtain clear cerebrospinal fluid (CSF); each resident had the necessary elements for puncture and CSF collection (gloves, scrubs, goggles, surgical drapes, antiseptic agents, puncture needles, and collection tubes). Experts in pediatrics and neurosurgery were asked for advice on how to prepare all components (theoretical module, videos and checklists), and previously-reported procedural guidelines and assessment lists were used. $^{7}$

Checklists used for assessing resident performance of LP on the phantom included the following dimensions: a) preparation of the infant for the procedure (environment and monitoring, use of analgesia, sepsis, glycemia monitoring, identification of materials); b) procedure (anatomical landmarks, biosafety measures, puncture and CSF collection); and c) communication with parents regarding the procedure.

In order to describe communicational skills, some of the components reported by the Kalamazoo Consensus Statement, ${ }^{8}$ which describe key elements of the physician-patient communication, were adapted. These include opening the discussion and introducing the physician, providing information, understanding parents' perspective, and ending the meeting (allowing time to make questions and reconfirming the message). Actors trained to simulate parents were available and had a script written for the context of the situation in the E.R. Department. Trainers observed pre-procedure conversations through a Gesell chamber and assessed selected items using a checklist. Finally, participants in the 2014 PR1 cohort (n: 54) were asked to report on their post-training experience performing an LP in a infant and to establish whether CSF was collected, if the experience was traumatic, or if there were any complications. The supervisor of the procedure reported on compliance with biosafety standards.

For each training session, the following was recorded: whether the resident had performed a prior LP, the level of confidence with the procedure (using a Likert scale, where 0 means "not at all confident" and 4, "highly confident") before and after the procedure, and the opinion on satisfaction with the educational experience (where 0 means "no satisfaction" and 4, "highly satisfactory").

FIGURE 1. Phantom used for lumbar puncture training

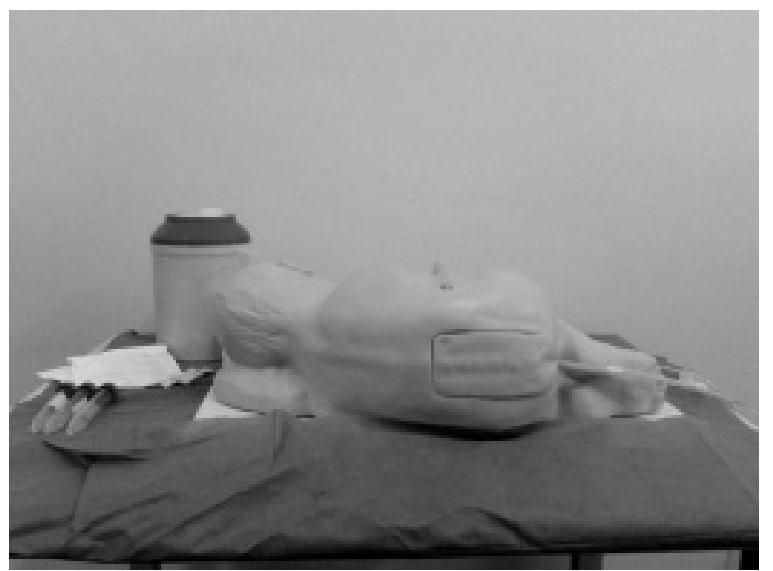


Four qualified trainers completed assessment checklists (compliant/non-compliant) at the simulation lab during each practice. Prior to this, communication with simulated parents was assessed through a Gesell chamber. Data were reported using central tendency and dispersion or frequency. Scores were described as sums and/or percentages of compliance.

One hundred twelve pediatric residents participated of training sessions; 65 of these (58\%) had never performed a lumbar puncture before, the rest had done it only once. All completed training. For the reported confidence level categories (Figure 2), 72\% (81/112) did not feel confident (at all or slightly) with performing an LP before receiving training. Confidence (feeling from mildly to highly confident) increased from $27 \%$ (31 residents) to 93\% (104 residents) after the simulated practice. Most participants considered that the experience was satisfactory or highly satisfactory.

Table 1 shows the results of the assessment checklist. Considering the sums of items complied with (for a maximum of 10), the average score was 7.01 (SD: 1.46) with a median of 7 (between 6 and 10), and no differences from the prior experience. Most items related to the procedure itself were complied with, but this is not the case of items related to preparation, which showed a $31 \%-67 \%$ compliance percentage. Table 2 shows a summary of results obtained in 98 of 112 residents in terms of communication.

Finally, 33 residents (61\%) completed a voluntary report submitted to the head of residents on how they felt with the LP experience on an actual patient following their training. Thirty residents $(91 \%)$ had managed to perform an LP satisfactorily, in five (15\%) they had been traumatic, and in $100 \%$ of cases, supervisors confirmed compliance with biosafety standards; no complications were recorded.

\section{DISCUSSION}

All PR1 (112) of two sequential cohorts completed the LP training program, which included simulation-based educational strategies. From the residents' perspective, the experience was feasible and satisfactory. This activity combined medical evidence-based information using online training strategies and supervised practice on phantoms in a contextualized setting. The possibility of learning in a lowstress environment seems adequate to increase confidence. The pre-training survey showed that PR1 had a low level of confidence with the procedure, even after learning the theory. Promes, et al., ${ }^{9}$ reported that less than $29 \%$ of

FIGURE 2. Residents' level of confidence with lumbar puncture before and after training; Likert scale: 0-4 (0: not at all confident; 4: highly confident)

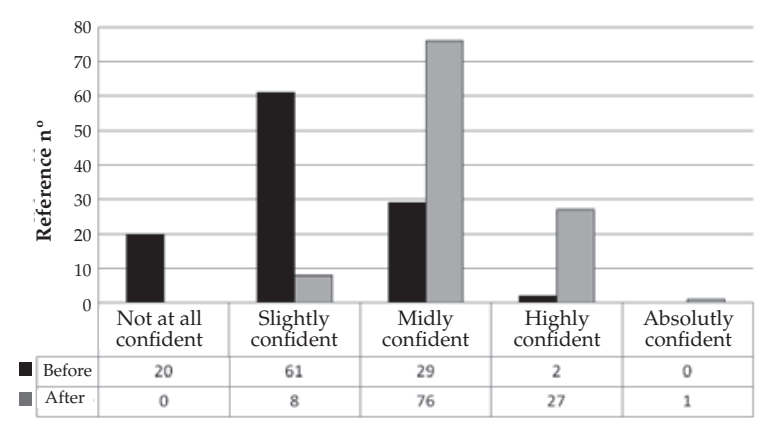

TABLE 1. Results of the checklist, preparation and performance of lumbar puncture during training of 112 residents

\begin{tabular}{lcc}
\hline & Compliant N (\%) & Non-compliant N (\%) \\
\hline Preparation for LP & & $73(65 \%)$ \\
1. Reviews monitoring and supply readiness for a possible resuscitation & $39(35 \%)$ & $77(68 \%)$ \\
2. Considersor prescribes analgesia & $35(31 \%)$ & $56(50 \%)$ \\
3. Requests or reviews blood glucose levels & $56(50 \%)$ & $40(36 \%)$ \\
4. $\quad$ Uses antiseptic measures correctly & $72(64 \%)$ & $37(33 \%)$ \\
5. Identifies materials for puncture & $75(67 \%)$ & $5(5 \%)$ \\
LP procedure & & $8(7 \%)$ \\
6. Establishes anatomicall and marks adequately & $107(95 \%)$ & $3(3 \%)$ \\
7. Performs puncture using an adequate technique & $104(93 \%)$ & $26(23 \%)$ \\
8. Collects CSF & $109(97 \%)$ & $9(8 \%)$ \\
9. Collects and sends CSF samples as per standards & $86(77 \%)$ & $103(92 \%)$ \\
10. Maintainsbio safety criteria & & \\
\hline
\end{tabular}

LP: lumbar puncture; CSF: cerebrospinal fluid. 
PR1 performed an LP during their medical graduate course; in our setting, such percentage is probably even lower. The change in confidence level after simulated training is consistent with what has been reported by other authors who have demonstrated this effect of simulation. ${ }^{10-12}$ The Simulation Center has been an optimal environment to conduct a contextualized practice, where procedure components preparation and information provided to simulated parents reached similar hierarchy for training as the procedure itself.

Assessing acquired skills requires reliable and valid measurement units. To this end, we used checklists consistent with training objectives and administered by trainers with experience in their use. Although most PR1 had good scores and there were no differences from the pre-training experience, it is worth noting that most procedure items were complied with, unlike items related to preparation, which achieved a $31 \%-67 \%$ compliance. Similarly, White, et al., ${ }^{13}$ reported that the most commonly overlooked steps are related to the preparation of elements to be used and/or documentation. Some specific aspects, such as an adequate pain management, require optimization. In our study, only $31 \%$ of residents considered or used analgesia correctly. In other groups, it has been reported that new E.R. residents completed only $57 \%$ of LP steps adequately, although $83 \%$ had previously performed this procedure. ${ }^{14}$ In relation to procedure reporting, items with a lower level of compliance were allowing parents to make questions and agreeing to let parents be present during LP. This is probably a reflection of guidelines conveyed by the hospital itself. Results reinforce the need to consider communication as a clinical competence that should be systematically taught and assessed, as has been currently accepted by different accreditation agencies. ${ }^{15}$ Simulation strategies using actors and interdisciplinary approaches allow, in this context, to adapt specific training programs aimed at teaching such competences.

A relevant aspect to be considered when measuring the impact of training is recognizing that assessing the simulated procedure does not ensure direct translation into clinical practice with patients. There is consensus regarding the search for direct assessment mechanisms in the clinical setting. ${ }^{16,17}$ In our study, the 2014 PR1 cohort reported on their first LP performed together with a responsible supervisor. This was a voluntary self-report and, in spite of its limitations (response accounted only for $61 \%$ ), our results show a $91 \%$ success rate, similar to what has been published by Kessler, et al., ${ }^{18}$ who reported a $94 \%$ success rate for CSF collection among participants trained using simulation, compared to $47 \%$ among those who received audiovisual information only. Other researchers have investigated the use of educational videos in relation to LP success ${ }^{19}$ and, although a significant improvement has been shown in certain technical aspects, no differences were observed and both groups had only a 50\% success. Finally, the implementation of procedural training integrating simulation strategies requires resources, adequate space, specific equipment and supplies, and qualified trainers with experience not only in the procedures, but also in using simulation strategies. In this experience, the availability of a simulation center in the hospital setting facilitated program implementation; however, the convenience of protected times and overall organization of activities in new settings, in the context of highly busy facilities, call for institutional commitment. In this context, aligning simulation with quality and patient safety is a strength that encourages the continuation of these efforts.

Our study has several limitations. It is worth noting that, even though we quantified improvement in confidence levels, there were no residents who did not receive training whose

TABLE 2. Results of communication of the procedure to simulated parents by 98 residents

\begin{tabular}{lcc}
\hline & Yes N (\%) & No N (\%) \\
\hline Introduces her/himself and greets parents & $75(76 \%)$ & $23(24 \%)$ \\
Uses an understandable language & $72(73 \%)$ & $26(27 \%)$ \\
Shows respect and empathy & $96(98 \%)$ & $2(2 \%)$ \\
Conveys confidence & $81(82 \%)$ & $17(18 \%)$ \\
Clearly explains the reason for the procedure and describes it & $58(59 \%)$ & $40(41 \%)$ \\
Allows parents to be present & $54(55 \%)$ & $44(45 \%)$ \\
Allows parents to make questions & $44(45 \%)$ & $54(55 \%)$ \\
Reassures that parents understand the information & $77(78 \%)$ & $21(22 \%)$ \\
\hline
\end{tabular}


performance with simulated practice and actual patients could be compared to that of participants. However, based on standardized checklists, our results are consistent with those reported in the literature and, in the future, may be compared to those of other institutions using different training models. Another limitation of this experience is related to the selected sample, because it corresponds to a single program in only one hospital, therefore reducing the possibility of extrapolating results. Finally, we also assessed certain communicational skills that, given their relevance, will require a specific and in-depth approach, in relation to both teaching modalities and assessment methods.

To sum up, a lumbar puncture training program integrating contextualized simulation strategies resulted feasible and highly satisfactory for residents, whose confidence improved and who gained more knowledge on such important and common pediatric procedure for those entering a pediatric training program.

It is obvious that newly graduated physicians face procedures for which they have not been trained and with which they feel little confidence. Such learning activities in a stressful environment and facing a child who requires a safe and effective procedure may be enhanced using new technologies and educational strategies. Their inclusion in the curricula, impact measurement, and resources necessary for their sustainability will be a challenge for those responsible for postgraduate training programs, who may not ignore that simulation does not replace supervised clinical practice, but improves learning of professional skills.

\section{CONCLUSION}

The simulated lumbar puncture training module allowed to teach this skill to PR1. At present, simulation has been introduced as an educational strategy in our program.

\section{REFERENCES}

1. Issenberg SB, McGaghie WC, Petrusa ER, Lee Gordon D, et al. Features and uses of high-fidelity medical simulations that lead to effective learning: a BEME systematic review. Med Teach 2005;27(1):10-28.

2. Bilotta FF, Werner SM, Bergese SD, Rosa G. Impact and implementation of simulation-based training for safety. Scientific World Journal 2013;2013:652956.

3. Wang EE, Quinones J, Fitch MT, Dooley-Hash S, et al. Developing technical expertise in emergency medicine. The role of simulation in procedural skill acquisition. Acad Emerg Med 2008;15(11):1046-57.
4. Auerbach M, Chang TP, Reid J, Quinones C, et al. Are pediatric interns prepared to perform infant lumbar punctures? A multi-institutional descriptive study. Pediatr Emerg Care 2013;29(4):453-7.

5. Argentina. Ministerio de Salud. Sistema Nacional de Residencias del Equipo de Salud. Marco de referencia para la formación en residencias médicas. Especialidad Pediatría. Buenos Aires, 2010. [Accessed on: August 22, 2014]. Availableat: http://www.msal.gov.ar/residencias/ images/stories/descargas/acreditaciones/adjuntos / pediatria.pdf.

6. Accreditation Council for Graduate Medical Education. ACGME Program Requirements for Graduate Medical Education in Pediatrics. [Accessed on: May 9, 2015]. Available at: http://www.acgme.org/acgmeweb/ Portals/0/PF Assets/Program Requirements/320_ pediatrics_2016.pdf.

7. Gerard JM, Kessler DO, Braun C, Mehta R, et al. Validation of global rating scale and checklist instruments for the infant lumbar puncture procedure. Simul Healthc 2013;8(3):148-54

8. Duffy FD, Gordon GH, Whelan G, Cole-Kelly K, et al. Assessing competence in communication and interpersonal skills: the Kalamazoo II report. Acad Med 2004;79(6):495-507.

9. Promes SB, Chudgar SM, Grochowski CO, Shayne P, et al. Gaps in procedural experience and competency in medical school graduates. Acad Emerg Med 2009;16(Suppl2):S58-62.

10. Hicks CM, Gonzalez R, Morton MT, Gibbons RV, et al. Procedural experience and comfort level in internal medicine trainees. J Gen Intern Med 2000;15(10):716-22.

11. Okuda Y, Bryson EO, DeMaria S Jr, Jacobson L, et al. The utility of simulation in medical education: what is the evidence? Mt Sinai J Med 2009;76(4):330-43.

12. Connick RM, Connick P, Klotsas AE, Tsagkaraki PA, et al. Procedural confidence in hospital-based practitioners: implications for the training and practice of doctors at all grades. BMC Med Educ 2009;9:2.

13. White ML, Jones R, Zinkan L, Tofil NM. Transfer of simulated lumbar puncture training to the clinical setting. Pediatr Emerg Care 2012;28(10):1009-12.

14. Lammers RL, Temple KJ, Wagner MJ, Ray D. Competence of new emergency medicine residents in the performance of lumbar punctures. Acad Emerg Med 2005;12(7):622-8.

15. Rider EA, Hinrichs MM, Lown BA. A model for communication skills assessment across the undergraduate curriculum. Med Teach 2006;28(5):e127-34.

16. Conroy SM, Bond WF, Pheasant KS, Ceccacci N. Competence and retention in performance of the lumbar puncture procedure in a task trainer model. Simul Healthc 2010;5(3):133-8.

17. Griswold S, Ponnuru S, Nishisaki A, Szyld D, et al. The emerging role of simulation education to achieve patient safety: translating deliberate practice and debriefing to save lives. Pediatr Clin North Am 2012;59(6):1329-40.

18. Kessler DO, Auerbach M, Pusic M, Tunik MG, et al. A randomized trial of simulation-based deliberate practice for infant lumbar puncture skills. Simul Healthc 2011;6(4):197203.

19. Srivastava G, Roddy M, Langsam D, Agrawal D. An educational video improves technique in performance of pediatric lumbar punctures. Pediatr Emerg Care 2012;28(1):12-6. 